\title{
Blind Estimation of Motion Blur Parameters For Image Deconvolution ${ }^{\star}$
}

\author{
João P. A. Oliveira, Mário A. T. Figueiredo, and José M. Bioucas-Dias \\ \{joao.oliveira, mario.figueiredo, jose.bioucas\}@lx.it.pt \\ Instituto de Telecomunicações, Instituto Superior Técnico, \\ Torre Norte, Piso 10, Av. Rovisco Pais, \\ 1049-001 Lisboa, PORTUGAL
}

\begin{abstract}
This paper describes an approach to estimate the parameters of a motion blur (direction and length) directly form the observed image. The motion blur estimate can then be used in a standard nonblind deconvolution algorithm, thus yielding a blind motion deblurring scheme. The estimation criterion is based on recent results about the general spectral behavior of natural images. Experimental results show that the proposed approach is able to accurately estimate both the length and orientation of motion blur kernels, even for small lengths which are traditionally difficult.
\end{abstract}

\section{Introduction}

In image deconvolution/deblurring problems, the goal is to estimate an original image $f=\{f(x, y), x=1, \ldots, N, y=1, \ldots, N\}$ from an observed version $g=$ $\{g(x, y), x=1, \ldots, N, y=1, \ldots, N\}$, assumed to have been produced according to

$$
g(x, y)=f(x, y) * h(x, y)+w(x, y),
$$

where $h(x, y)$ is the blur point spread function (PSF), $\{n(x, y), x=1, \ldots, N, y=$ $1, \ldots, N\}$ is a set of independent samples of zero-mean Gaussian noise of variance $\sigma^{2}$, and $*$ denotes the discrete two-dimensional (2D) convolution,

$$
f(x, y) * h(x, y) \equiv \sum_{u} \sum_{v} f(u, v) h(x-u, y-v) .
$$

After collecting the elements of $f, g$, and $n$ into vectors $\mathbf{f}, \mathbf{g}, \mathbf{n} \in \mathbb{R}^{N \times N}$, usually in lexicographic order, (1) can be written in vector notation as

$$
\mathrm{g}=\mathbf{H x}+\mathbf{w},
$$

where $\mathbf{H}$ is a matrix representing the blur operator. This makes clear that (1) is a particular case of the general problem of signal/image restoration from linear and noisy observations. For most non-trivial blur kernels, the corresponding matrix

\footnotetext{
* This work was supported by Fundação para a Ciência e Tecnologia, under project
} POSC/EEA-CPS/61271/2004. 
is severely ill-conditioned, or even singular, making deconvolution an ill-posed problem.

It's well known that the ill-posed nature of image deconvolution demands some kind of regularization to be used. Among the state of the art methods, we find those based on total variation (TV) regularization (see $[3,4,8]$ and further references therein) which favors images of bounded variation, without penalizing possible discontinuities, as well as wavelet-based methods $[2,9,10]$, which also provide regularization without overly penalizing image discontinuities.

In standard deconvolution problems, it's assumed that $\mathbf{H}$ (equivalently $h$ ) is fully and exactly known. In blind deconvolution, the goal is to deblur an image with (total of partial) lack of knowledge about the blurring operator [13, 14]. It's clear that blind deconvolution is significantly more difficult than its non-blind counterpart [1]. The problem is now ill-posed both with respect to the unknown original image and to the blur operator. Simply put (and because convolution can be expressed as a product in the Fourier domain), blind deconvolution can be seen as the problem of recovering two functions from their product; a clearly hopeless goal, in the absence of some rather strong assumptions or prior knowledge about the underlying image and blur. Assumptions about the blur PSF which have been used are positiveness, known shape (e.g., Gaussian blur) or known support.

There are essentially two alternative approaches to blind deconvolution: (i) simultaneously estimate the image and the blur [1,7]; (ii) perform a previous step of blur estimation and then feed this blur estimate to a classical non-blind image deblurring algorithm [6]. In this paper, we introduce a blur estimation technique to be used in an approach of type (ii). More specifically, we introduce a method to estimate the parameters of a "motion blur" from the noisy blurred image, with weak assumptions on the underlying original image.

The particular class of blur operators herein considered, known as motion blur, arises when there is relative motion between the acquisition device (i.e., the camera) and the scene being acquired. For simplicity, we assume that the camera is moving with constant speed and direction, with respect to the scene. The resulting blur kernel/filter has a linear support in the spacial domain and a sinclike (recall that $\operatorname{sinc}(x)=\sin (x) / x$ ) behavior in the Fourier domain, leading to well pronounced valleys in the logarithm of the magnitude of the spectrum of the observed image. The method proposed in this paper, exploits the identification of the parameters characterizing this sinc-like behavior (namely, the orientation and period, which correspond to the orientation and length of the blur kernel) using the well known Radon transform [5]. The proposed algorithm is able to accurately estimate the motion blur parameters (orientation and length) for motion blurs with lengths as short as three pixels.

This paper is organized as follows. In Section 2 we define the motion blur and corresponding parameters for the continuous and the discrete cases. We also define the blur kernel structure used in this work. In Section 3.1, we review some of the state of the art methods and present the proposed algorithm. In section 4 we report the performance of the proposed method and in the final section we draw some conclusions. 


\section{Motion Blur Parameters}

In the continuous case, the motion blur PSF is characterized by a normalized delta function, supported on a line segment with length $L$ and an angle $\theta$ (for example, with respect to the horizontal). The angle gives the direction of motion, and the length $L$ is proportional to the motion speed. To deal with digital images, we need a discrete version of this motion blur, defined on the discrete pixel grid. To produce a straight line segment on a digital grid, we use a standard algorithm (well known in computer graphics): the digital differential analyzer (DDA) [11]. The length $L$ is assumed to be, for all angles, the number of pixels, and is directly proportional to the motion speed. As can be easily verified, there is no way to exactly produce lines with one pixel width in all possible directions.

One big limitation of this DDA-based discrete PSF approximation, or any discrete representation in general, is that it cannot distinguish between two blur kernels with nearby angles. This effect is stronger for shorter motion blur kernels. For example, a three pixels long kernel can only have five different directions between $0^{\circ}$ and $90^{\circ}$. With all these assumptions and limitations in hand, our goal is to estimate the angle of motion and the motion speed parameters from an observed image, without knowledge of the underlying original image.

\section{Estimation of Motion Blur Parameters}

\subsection{Natural Image Models}

Let us denote as $F(\xi, \eta)$ the 2D Fourier transform of a natural image $f(x, y)$. As pointed out in [6], the behavior of $\log |F(\xi, \eta)|$ along lines $\eta=\xi \tan \theta$ in the $(\xi, \eta)$ plane, is roughly the same for most natural images. While local behavior may be irregular, the coarse/global behavior is essentially monotonically decreasing with $\xi$. In [6], the approximate model $\log |F(\xi, \xi \tan \theta)| \simeq-a|\xi|^{b}$, with $a, b>0$, was proposed. Although $a$ and $b$ can vary for different images, this global behavior is approximately true, regardless of the considered angle.

If we take the Fourier transform of equation (1), we obtain

$$
G(\xi, \eta)=F(\xi, \eta) H(\xi, \eta)+W(\xi, \eta),
$$

where $F, G, H$, and $W$ are the Fourier transforms of $f, g, h$, and $w$, respectively. As is common in deconvolution problems, assuming the noise is weak, we have

$$
\log |G(\xi, \eta)| \approx \log |F(\xi, \eta) H(\xi, \eta)|
$$

and so the coarse behavior of the $G(\xi, \eta)$ depends essentially on the product $F(\xi, \eta) H(\xi, \eta)$. Since the coarse behavior $F(\xi, \eta)$ along lines $\eta=\xi \tan \theta$ in the $(\xi, \eta)$ plane is approximately independent of $\theta$, the sinc-like structure of $H(\xi, \eta)$ is preserved in $G(\xi, \eta)$. In the presence of noise, the zeros of this sinc function become local minima. In order to capture these coarse behaviors, we will use the Radon transform (RT) of $\log |G(\xi, \eta)|$, as described in the following subsections. 


\subsection{The Radon Transform}

Let us briefly recall the definition of Radon transform (RT) of a real-valued function $\phi(x, y)$ defined on $\mathbb{R}^{2}$, at angle $\theta$ and distance $\rho$ from the origin:

$$
\mathcal{R}(\phi, \rho, \theta)=\int_{-\infty}^{\infty} \int_{-\infty}^{\infty} \phi(x, y) \delta(\rho-x \cos \theta-y \sin \theta) d x d y
$$

where $\delta$ denotes the Dirac delta function. Equivalently,

$$
\mathcal{R}(\phi, \rho, \theta)=\int_{-\infty}^{\infty} \phi(\rho \cos \theta-s \sin \theta, \rho \sin \theta+s \cos \theta) d s .
$$

The Radon transform $\mathcal{R}(\phi, \rho, \theta)$ is equal to the integral of $\phi$ along a line that forms an angle $\theta$ with the $x$-axis and is at a distance $\rho$ from the origin [5].

\subsection{Angle Estimation}

The RT has been recently proposed for motion blur estimation in [12, 15]. In [15], the angle estimate is the one for which the maximum of the RT occurs; naturally, this only works for rather long blurs, so that the image gets flat, leading to a maximum of the RT. In [12], the angle estimate is that for which $\mathcal{R}(\phi, \rho, \theta)$, as a function of $\rho$, has highest entropy. The authors claim to have a problem at $45^{\circ}$ (due to the finite support of the images): at this angle, the entropy is maximum because the length of the integral is maximum, thus picking up the largest amount of noise (according to the authors' explanation). To circumvent this problem, they normalize the RT of the image with the RT of a matrix of 1 's of the same dimension as the image. However, this explanation is not true; the true reason is related to the implementation of RT for digital images.

Our approach circumvents these limitations and allows excellent angle estimation performance, even for very short lengths. We use an exact RT for digital images, the details of which can be found in [16]. As explained above, for natural images, $\log |G(\xi, \eta)|$ has an approximate coarse behavior along radial lines, independent of the angle; thus the RT obtained by integrating on similar intervals, that is, with the same area for any direction, will also be approximately equal. Accordingly, instead of computing the RT for the whole image, we integrate in the maximum inscribed square,

$$
\mathcal{R}_{d}(f, \rho, \theta)=\int_{-d}^{d} f(\rho \cos \theta-s \sin \theta, \rho \sin \theta+s \cos \theta) d s,
$$

with $d=\frac{\sqrt{2}}{2} N$. This RT of $\log |G(\xi, \eta)|$ has approximately the same variance, independently of $\theta$, thus avoiding the angle-dependence problem pointed out and addressed in [16]. Consequently, the variance of $\mathcal{R}_{d}(\log |G(\xi, \eta)|, \rho, \theta)$, as a function of $\theta$, depends fundamentally on the orientation of the blur kernel.

The observations made in the previous paragraph lead to our proposed estimate for the angle of the motion blur kernel, which is given by

$$
\widehat{\theta}=\arg \max _{\theta} \operatorname{var}\left\{\mathcal{R}_{d}(\log |G(\xi, \eta)|, \rho, \theta)\right\}
$$

where $\operatorname{var}\{\}$ is the variance of the set of values obtained by varying $\rho$. 


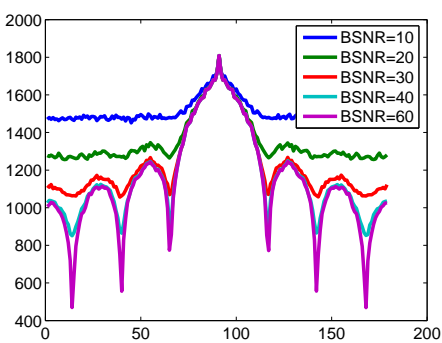

a) Angle $=0^{\circ}$

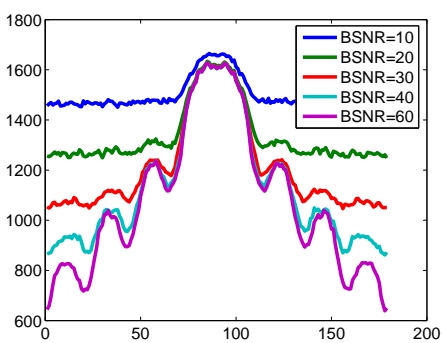

b) Angle $=60^{\circ}$

Fig. 1. Radon transform of the blurred cameraman $(L=10)$.

\subsection{Length Estimation}

Once we have $\widehat{\theta}$, the estimate of the motion blur direction, we proceed to estimate the length of the motion blur kernel. Given that the sinc-like behavior is preserved in the RT along $\widehat{\theta}$, we base the estimation of the blur length on $\mathcal{R}_{d}(\log |G(\xi, \eta)|, \rho, \widehat{\theta})$. This line of attack is also followed in [15] using fuzzy sets and in [12] exploiting cepstral features. Figure 1 illustates this behaviour. The RT of the "cameraman" image, for angles $\theta=0^{\circ}$ and $\theta=60^{\circ}$ and blur length $L=10$ exibits a clear sinc-like pattern.

In the present work, we propose an heuristic algorithm simpler than those introduced in $[15,12]$, yet accurate. Let's denote $\Pi(\omega)=\mathcal{R}_{d}(\log |G(\xi, \eta)|, \omega, \widehat{\theta})$. From the above rationale, we conclude that the minima of $\Pi(\omega)$ are the minima of the Fourier transform of a rectangular pulse of size $L$. Let's then assume for a while that $\Pi(\omega)$ is indeed the Fourier transform of a rectangular pulse of length $L_{S}$, i.e,

$$
\Pi(\omega)=e^{j \psi(\omega)} \frac{\sin \left(\frac{\omega L_{S}}{2}\right)}{\sin \left(\frac{\omega}{2}\right)} .
$$

Our goal is to find $L_{S}$ by seeking for the first positive zero of $\Pi(\omega)$ given by $\omega_{0}=\frac{2 \pi}{L_{S}}$. Since we have determined $\mathcal{R}_{d}$ based on an $M$ point FFT, we approximate $\omega_{0}=\frac{2 \pi}{L_{S}}$ with the frequency $\frac{2 \pi}{M} i$ corresponding to the minima of the FFT magnitude. We have then $\widehat{L}_{S}=\operatorname{round}(M / i)$.

Algorithm 1 shows the pseudo-code for the estimation of the length $\widehat{L}$. The lines 2, 3, and 4 implement a robust estimator of the first minimum. Line 5 converts distance to number of pixels. Figure 2 illustrates the working of Algorithm 1 with two different RTs.

\section{Experimental Results}

In this section we evaluate the performance of the proposed algorithm in terms of the root mean squared error (RMSE) of the estimated parameters. We considered a set of 7 well known images: cameraman, Lena, Barbara, boats, mandrill, 


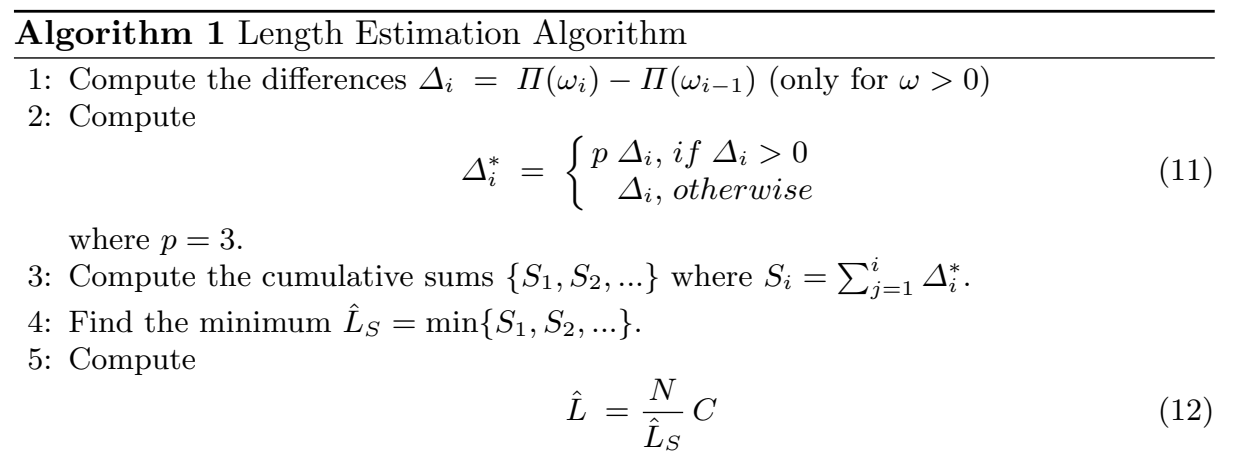

where $C$ is correction term is given by

$$
C=\left\{\begin{array}{l}
\cos (\hat{\theta}), \text { if }|\hat{\theta}| \leq \pi / 4 \\
\sin (\hat{\theta}), \text { if } \pi / 4<|\hat{\theta}| \leq 3 \pi / 4
\end{array}\right.
$$

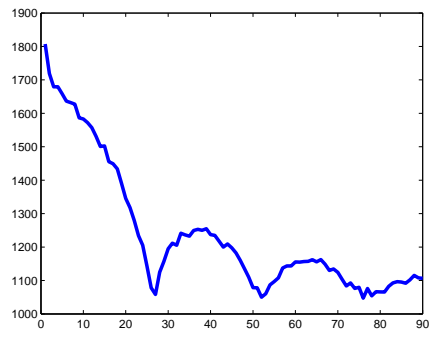

a) RT, $B S N R=30 d B$

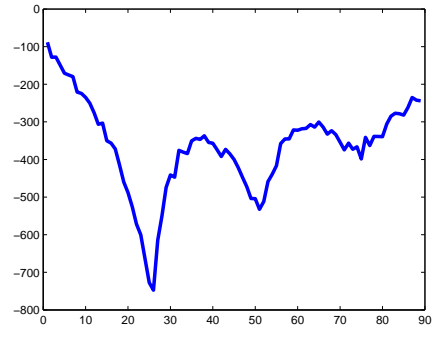

b) Cummulative sums $S_{i}, B S N R=30 d B$

Fig. 2. Radon transform and cummulative sums $S_{i}$ for motion blur of length $=10$ pixels, angle $=0^{\circ}$

goldhill and peppers, all of size 256 by 256 pixels. RMSE is computed based on 70 runs, 10 for each image.

\subsection{Angle Estimation}

The use of previously introduced DDA-based discrete PSF approximation makes it impossible to distinguish two kernels with nearby angles. Given a blur length $L$, we compute the intervals and respective middle angle, $\theta_{m}(L)$, that leads to the same blur kernel. For each test angle, we choose the closest $\theta_{m}(L)$ and use this value in the simulations. We proceed in the same way for the estimated angle, which leads to the error defined by

$$
\text { error }=\hat{\theta}_{m}(L)-\theta_{m}(L) \text {. }
$$

Since we can not evaluate all possible scenarios, we considered six different motion blur lengths, $L \in\{4,12,20,24,32,40\}$ pixels, with different blur signal to noise ratio $\mathrm{BSNR} \in\{10,40\}(\mathrm{dB})$. The obtained results are shown in figure (3). 


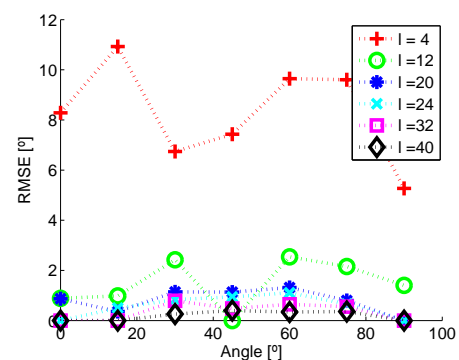

a) $B S N R=10 \mathrm{~d} B$

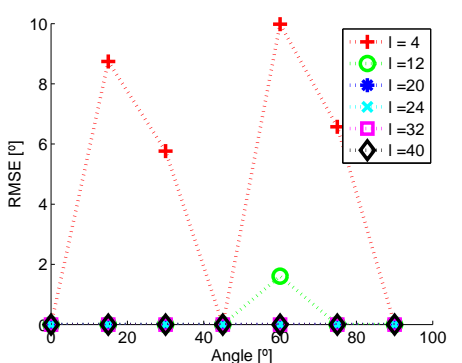

b) $S$ function, $B S N R=40 d B$

Fig. 3. Estimation angle error for different BSNR and different blur length.

The results obtained clearly show the accuracy of the proposed algorithm. The worst errors are obtained for small blur kernels, as we would expect.

\subsection{Length Estimation}

In a similar way, we considered four different angles, $\theta \in\left\{0^{\circ}, 15^{\circ}, 30^{\circ}, 45^{\circ}\right\}$, and two different BSNR, 15 and $40(\mathrm{~dB})$. The obtained results are shown in figure 4.

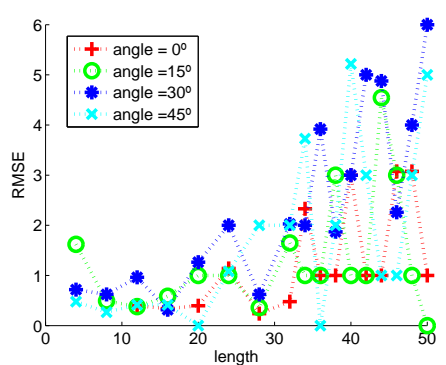

a) $B S N R=15 d B$

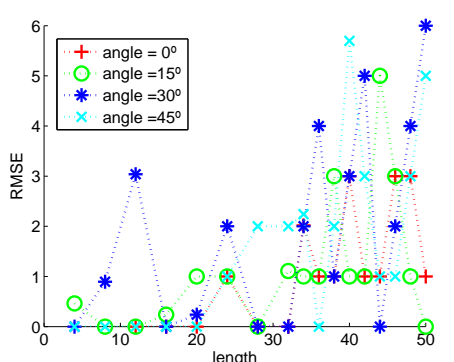

b) $B S N R=40 d B$

Fig. 4. Estimation length error for different BSNR and different blur angle.

The results again show the accuracy of the proposed algorithm, even in the worst BSNR. For large blur lengths, the errors are bigger, given that the first zero of $\Pi(\omega)$ is close to the origin and $\hat{L}$ is inversely proportional to this corresponding frequency. Then small errors in this location are very amplified. For short blurs, the proposed algorithm fails when the BSNR is low. In this situation, the RT is too noisy, and the heuristic value of $p$ used in Algorithm 1 leads to poor estimate.

\section{Conclusions}

The paper introduced a robust algorithm to infer motion blur parameters, namely the motion direction and the motion length. The angular quasi-invariance of 
natural images spectrum was exploited in the Radom transform domain. The effectiveness of the method was illustrated in a set of experiments.

\section{References}

1. L. Bar, N. Sochen and N. Kiryati, "Variational pairing of image segmentation and blind restoration" , Proc. 8th European Conference on Computer Vision ECCV'2004, pp. 166-177, Prague, Czech Republic, 2004.

2. J. Bioucas-Dias, "Bayesian wavelet-based image deconvolution: a GEM algorithm exploiting a class of heavy-tailed priors," IEEE Transactions on Image Processing, vol. 15, pp. 937-951, 2006.

3. J. Bioucas-Dias, M. Figueiredo, J. Oliveira, "Total-variation image deconvolution: A majorization-minimization approach", IEEE International Conference on Acoustics, Speech, and Signal Processing - ICASSP'2006, Toulouse, France, 2006.

4. J. Bioucas-Dias, M. Figueiredo, J. Oliveira, "Adaptive Bayesian/total-variation image deconvolution: A majorization-minimization approach", European Signal Processing Conference - EUSIPCO'2006, Florence, Italy, 2006.

5. R. Bracewell, Two-Dimensional Imaging, Prentice Hall, 1995.

6. A. Carasso, "Direct blind deconvolution", SIAM Journal of Applied Mathematics, vol. 1, pp. 1980-2007, 2001.

7. T. Chan and C. Wong. "Total variation blind deconvolution", IEEE Transactions on Image Processing, vol. 7, pp. 370-375, 1998.

8. P. Combettes and J. Pesquet, "Image deconvolution with total variation bounds", Proceedings of the Seventh International Symposium on Signal Processing and Its Applications, vol. 1, pp. 441-444, Paris, France, 2003.

9. M. Figueiredo and R. Nowak, "An EM algorithm for wavelet-based image restoration," IEEE Trans. on Image Processing, vol. 12, no. 8, pp. 906-916, 2003.

10. M. Figueiredo and R. Nowak, "A bound optimization approach to wavelet-based image deconvolution," IEEE Intern. Conf. on Image Processing - ICIP'05, Genoa, Italy, 2005.

11. S. Harrington, Computer Graphics: A Programming Approach, McGraw-Hill, 1985.

12. F. Krahmer, Y. Lin, B. McAdoo, K. Ott, J. Wang, D. Widemannk, and B. Wohlberg, "Blind image deconvolution: motion blur estimation", Technical Report, Institute of Mathematics and its Applications, University of Minnesota, 2006.

13. D. Kundur and D. Hatzinakos, "Blind image deconvolution", Signal Processing Magazine, vol. 13, no. 3, pp. 43-64, 1996.

14. D. Kundur and D. Hatzinakos, "Blind image deconvolution revisited", Signal Processing Magazine, vol. 13, no. 6, pp. 61-63, 1996.

15. M. Moghaddam and M. Jamzad, "Motion blur identification in noisy images using fuzzy sets", Proceedings of the Fifth IEEE International Symposium on Signal Processing and Information Technology, pp. 862-866, 2005.

16. J. Oliveira, "Implementation of an exact Radon transform", Technical Report, Instituto de Telecomunicações, Lisboa, 2006. 